\title{
Uric acid and xantine-oxidase inhibitors in patients with gout: A re-assessment and an update
}

\author{
Claudio Borghi ${ }^{1}$, Krzysztof Narkiewicz ${ }^{2}$, Giuseppe Mancia ${ }^{3}$ \\ ${ }^{1}$ Department of Medical and Surgical Sciences, University of Bologna, Italy \\ ${ }^{2}$ Department of Hypertension and Diabetology, Medical University of Gdansk, Poland \\ ${ }^{3}$ Università Milano-Bicocca, Milan, Italy
}

Gout is a common inflammatory disease in which pathogenesis is almost entirely supported by the deposition of elevated levels of urate crystals in many different tissues, particularly the joints and the kidneys [1]. Gout is also associated with an increase in risk of cardiovascular disease (in particular coronary artery disease) which significantly contributes to higher mortality and morbidity [2]. The clinical manifestations of gout are usually heralded by an asymptomatic increase in plasma levels of uric acid, this must be considered as a typical biomarker of the disease. An individuals' risk of gout is proportional to absolute circulating levels of uric acid and increases progressively with the number of attacks and poor control of uric acid levels [3]. Froma biochemical point of view, uric acid is the final step of a biochemical cascade aimed at the degradation of purines [4] and is largely based on the activation of the enzyme xantine-oxido-reductase (XOR) that catalyzes the last two steps of the reaction, i.e. those leading to the production of uric acid. This can explain why the treatment of gout and the prevention of its clinical complications are largely based on the administration of drugs that reduce the levels of serum uric acid through the inhibition of the XOR ("XO-inhibitors").

In current clinical practice, allopurinol and febuxostat are the two drugs commonly used to inhibit XOR, leading to a significant reduction of serum levels of uric acid and a decrease in the recurrence of gout attacks and flares [5]. They are recommended as the first-line drugs by all major guidelines regarding the management of inflammatory diseases, it is also mentioned that these two drugs have been shown to exert a preventive effect on cardiovascular disease and may thus be considered in the management of patients with gout in whom cardiovascular prevention is a fundamental therapeutic goal, such as when cardiovascular risk is high or very high.

In a recent issue of the Cardiology Journal [6], a position paper was published dealing with the use of XO-inhibitors in patients with gout and summarized most of the available information about their efficacy and safety. This paper reviewed data showing that febuxostat provides a potent and highly selective inhibition of $\mathrm{XO}$, and thus greater hypouricemic activity vs the commonly used doses of allopurinol [7]. It was also mentioned, however, that results of the CARES study [8] reported an increased risk of cardiovascular death in patients treated with febuxostat and subsequently led some regulatory authorities to issue a cautionary statement on the widespread use of the drug. It is herein emphasized that the limitations of the CARES study (which extend to trial design) as well as evidence presented after its publication largely remove the arguments against the use of febuxostat in patients with gout. As mentioned in the paper [6] the CARES study did not include a non-actively treated or placebo control group, which importantly limits the interpretation of its findings. Furthermore, the study had been carried out in patients with moderate-to-severe obesity and had a history of a major cardiovascular event, namely a very high cardiovascular risk population that was far from the average population of patients suffering from gout in clinical practice. Finally, and most importantly, a recently published metaanalysis of 35 studies did not show a significant

Address for correspondence: Prof. Krzysztof Narkiewicz, Department of Hypertension and Diabetology, Medical University of Gdansk, ul. Dębinki 7c, 80-952 Gdańsk, Poland, e-mail: knark@gumed.edu.pl

Received: 19.01.2019 Accepted: 19.01.2019 
Table 1. Number of adverse events in chronic kidney disease patients treated with febuxostat or placebo. FEATHER Study (from [11] modified).

\begin{tabular}{lccc}
\hline Events & Febuxostat $(\mathbf{n}=\mathbf{2 2 2})$ & Placebo $(\mathbf{n}=219)$ & $\mathbf{P}$ \\
\hline Renal dysfunction & 9 & 19 & 0.08 \\
Cardiovascular disorders & 4 & 9 & 0.2 \\
Stroke & 1 & 2 & 0.6 \\
Other cardiovascular & 3 & 10 & 0.08 \\
Blood pressure changes & 5 & 8 & 0.5 \\
Arrhythmias & 4 & 4 & 0.9 \\
Total & $26(11.7 \%)$ & $62(28.3 \%)$ & - \\
\hline
\end{tabular}

difference between febuxostat and allopurinol for the risk of cardiovascular events $(\mathrm{p}=0.37)$ [9]. Thus, there appears to be no reason for concern about the use of febuxostat in the general population affected by gout, strictly limiting the debated question about a highly selected group of patients in whom gout, complicated by obesity and very high cardiovascular risk. Even in these patients, however, a favorable cardiovascular effect of febuxostat cannot be excluded because in the CARES study, the lack of a placebo arm did not allow a provision for information on the overall cardiovascular effects of reduction vs. no reduction of uric acid.

These re-assuring conclusions have been strongly supported by the studies presented during an international meeting on uric acid and XO-inhibitors held in Italy under the auspices of the European Society of Hypertension in November 2018. In the FREED study (Kojima S, ESC Congress 2018) [10], over 1000 elderly patients with elevated serum uric acid levels (between 7.0 and $9.0 \mathrm{mg} /$ day) and one or more risk factors for cerebral, cardiovascular, or renal disease were treated with febuxostat (up to $40 \mathrm{mg}$ daily) or been given no or other hypouricemic drugs (100 $\mathrm{mg}$ daily of allopurinol). After 3 years, a $25 \%$ reduction in the risk of a composite of death for any cause, cerebrovascular disease, non-fatal coronary disease, heart failure requiring hospitalization, atherosclerotic disease requiring treatment, renal impairment, and atrial fibrillation was observed in febuxostat compared to the non-febuxostat group. Febuxostat also prevented the development and progression of chronic kidney disease. In the multicenter FEATHER study, patients with different degrees of chronic kidney disease $(n=467)$ were randomly assigned to receive febuxostat or placebo for 108 weeks. Treatment with febuxostat did not significantly prevent the decline in kidney function in patients with stage 3 chronic kidney disease and asymptomatic high uric acid [11]. However, in a pre-specified subgroup analysis on patients with different degrees of renal dysfunction (presence/ /absence of proteinuria and different levels of serum creatinine) febuxostat exhibited a favorable effect when renal function was better preserved. In addition, a detailed analysis of the incidence of severe adverse effects showed that the drug was associated with a lower incidence of major cardiovascular and renal complications (Table 1), clearly supporting the adequate safety profile of the drug. Finally, a recent comparative analysis was carried out on a database of United States Medicare (2008-2013), using propensity score matching (ratio 1:3) to limit confounding [12]. The analysis included 99,744 elderly (age $>65$ years) patients with gout who initiated urate lowering treatment with febuxostat or allopurinol. The incidence of the primary outcome (hospitalization of myocardial infarction or stroke) per 100 person-years was 3.43 and 3.36, respectively in patients initiating treatment with febuxostat and allopurinol (hazard ratio: $1.01 ; 95 \%$ confidence interval: 0.94-1.08). Subgroup and sensitivity analyses consistently showed a similar cardiovascular risk in both groups, with no overall difference in risk of myocardial infarction, stroke, new-onset heart failure, coronary revascularization, or all-cause mortality as well. These results on populations are more clearly representative of patients with gout, candidates to which hypouricemic treatment in clinical practice clearly support the conclusions about the general safety of febuxostat, the only residual query remaining were small subgroups of "CARES" patients where the impact of hypouricemic treatment must be addressed by studies with a better controlled design. This is in line with a statement recently published in Circulation by W.B. White, the leading author of the CARES paper: "The unexpected finding of an increase in cardiovascular mortality with no 
differences in any non-fatal cardiovascular event in CARES requires more research in the assessment of cardiovascular safety of xanthine oxidase inhibitors" [13].

\section{Conflict of interest: None declared}

\section{References}

1. Kuo CF, Grainge MJ, Zhang W, et al. Global epidemiology of gout: prevalence, incidence and risk factors. Nat Rev Rheumatol. 2015; 11(11): 649-662, doi: 10.1038/nrrheum.2015.91, indexed in Pubmed: 26150127.

2. Johnson RJ, Bakris GL, Borghi C, et al. Hyperuricemia, Acute and Chronic Kidney Disease, Hypertension, and Cardiovascular Disease: Report of a Scientific Workshop Organized by the National Kidney Foundation. Am J Kidney Dis. 2018; 71(6): 851-865, doi: 10.1053/j.ajkd.2017.12.009, indexed in Pubmed: 29496260.

3. Bardin T, Richette P. Definition of hyperuricemia and gouty conditions. Curr Opin Rheumatol. 2014; 26(2): 186-191, doi: 10.1097/ BOR.0000000000000028, indexed in Pubmed: 24419750.

4. Johnson RJ, Titte S, Cade JR, et al. Uric acid, evolution and primitive cultures. Semin Nephrol. 2005; 25(1): 3-8, indexed in Pubmed: 15660328.

5. Richette P, Doherty M, Pascual E, et al. 2016 updated EULAR evidence-based recommendations for the management of gout. Ann Rheum Dis. 2017; 76(1): 29-42, doi: 10.1136/annrheumdis-2016-209707, indexed in Pubmed: 27457514.

6. Borghi C, Tykarski A, Widecka K, et al. Expert consensus for the diagnosis and treatment of patient with hyperuricemia and high cardiovascular risk. Cardiol J. 2018; 25(5): 545-563, doi: 10.5603/ /CJ.2018.0116, indexed in Pubmed: 30394510.
7. Borghi C, Perez-Ruiz F. Urate lowering therapies in the treatment of gout: a systematic review and meta-analysis. Eur Rev Med Pharmacol Sci. 2016; 20(5): 983-992, indexed in Pubmed: 27010159.

8. White WB, Saag KG, Becker MA, et al. CARES Investigators. Cardiovascular safety of febuxostat or allopurinol in patients with gout. N Engl J Med. 2018; 378(13): 1200-1210, doi: 10.1056/ /NEJMoa1710895, indexed in Pubmed: 29527974.

9. Richette P, Latourte A, Bardin T. Cardiac and renal protective effects of urate-lowering therapy. Rheumatology (Oxford). 2018; 57(suppl_1): i47-i50, doi: 10.1093/rheumatology/kex432, indexed in Pubmed: 29272510.

10. Kojima S, Matsui K, Ogawa $\mathrm{H}$, et al. Rationale, design, and baseline characteristics of a study to evaluate the effect of febuxostat in preventing cerebral, cardiovascular, and renal events in patients with hyperuricemia. J Cardiol. 2017; 69(1): 169-175, doi: 10.1016/j.jjcc.2016.02.015, indexed in Pubmed: 27005768.

11. Kimura K, Hosoya T, Uchida S, et al. FEATHER Study Investigators. Febuxostat Therapy for Patients With Stage 3 CKD and Asymptomatic Hyperuricemia: A Randomized Trial. Am J Kidney Dis. 2018; 72(6): 798-810, doi: 10.1053/j.ajkd.2018.06.028, indexed in Pubmed: 30177485.

12. Zhang M, Solomon DH, Desai RJ, et al. Assessment of cardiovascular risk in older patients with gout initiating febuxostat versus allopurinol. Circulation. 2018; 138(11): 1116-1126, doi: 10.1161/CIRCULATIONAHA.118.033992, indexed in Pubmed: 29899013.

13. White WB. Gout, xanthine oxidase inhibition, and cardiovascular outcomes. Circulation. 2018; 138(11): 1127-1129, doi: 10.1161/CIRCULATIONAHA.118.036148, indexed in Pubmed: 30354393. 